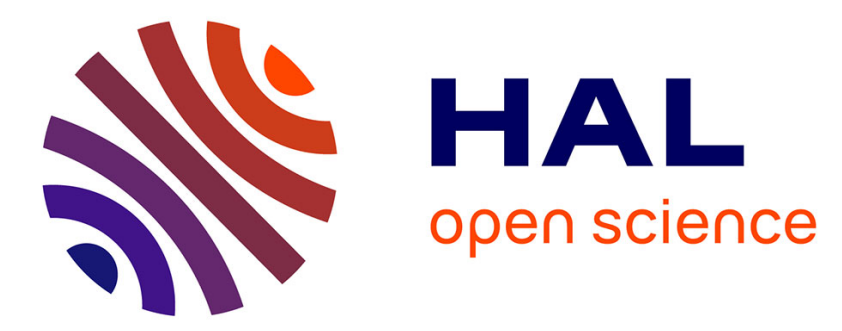

\title{
Thermal properties of mid-infrared colloidal quantum dot detectors
}

\author{
Emmanuel Lhuillier, Sean E. Keuleyan, Rekemeyer Paul, Philippe
}

Guyot-Sionnest

\section{- To cite this version:}

Emmanuel Lhuillier, Sean E. Keuleyan, Rekemeyer Paul, Philippe Guyot-Sionnest. Thermal properties of mid-infrared colloidal quantum dot detectors. Journal of Applied Physics, 2011, 110 (3), pp.033110. 10.1063/1.3619857 . hal-01438574

\section{HAL Id: hal-01438574 \\ https://hal.science/hal-01438574}

Submitted on 25 Aug 2020

HAL is a multi-disciplinary open access archive for the deposit and dissemination of scientific research documents, whether they are published or not. The documents may come from teaching and research institutions in France or abroad, or from public or private research centers.
L'archive ouverte pluridisciplinaire HAL, est destinée au dépôt et à la diffusion de documents scientifiques de niveau recherche, publiés ou non, émanant des établissements d'enseignement et de recherche français ou étrangers, des laboratoires publics ou privés. 


\section{Abstract}

James Franck Institute, 929 E. $57^{\text {th }}$ Street, The University of Chicago, Chicago, Illinois 60637,

$U S A$

Keywords: Quantum dot, infrared, HgTe.

PACS: 85.35.Be, 85.60.Gz

HgTe colloidal quantum dot films are studied for photodetection over the 3-5 $\mu \mathrm{m}$ atmospheric 9 transparency window. The temperature dependence of the conductivity indicates that the material behaves approximately as an intrinsic semiconductor. In photoconduction, the responsivity can be as high as several hundred $\mathrm{mA} \mathrm{W}^{-1}$ at room temperature. The dark current presents $1 / f$ noise which is larger than for homogeneous conductors, and this noise decreases with temperature. A specific detectivity of $2 \times 10^{9}$ Jones is obtained for a sample with a 6 micron cut-off wavelength at

4 130K. These values are obtained for the thickest films studied $(\sim 400 \mathrm{~nm})$ and whose thicknesses

5 are still much less than the optical absorption length. The time response can be faster than 100 nanoseconds.

\footnotetext{
*To whom correspondence should be sent : pgs@uchicago.edu
} 


\section{INTRODUCTION}

19 Colloidal quantum dots (CQD) have been studied over more than two decades, and they have 20 potential for various optoelectronic applications ${ }^{1}$. While sharing the advantages of liquid

21 processing with organic molecular compounds, they have a very unique advantage in the infrared 22 due to weaker vibrational absorption and well defined infrared electronic absorption. They have 23 already been extensively investigated in the near-IR for photodetection, ${ }^{2,3,4,5,6}$. $\mathrm{PbS}$ and $\mathrm{PbSe}$ 24 quantum dots are potential materials in the near- $\operatorname{IR}^{7,8,9,10}$. For longer wavelengths, $\operatorname{HgTe}$ should 25 be a better choice since it is a gapless semiconductor ${ }^{5,11}$. As CQD, this material had already been

26 shown to function well for near infrared photodetection ${ }^{12,13}$ and we recently extended the 27 material's response into the mid-IR. ${ }^{14}$

28 CQD may address current demand for a decrease of the cost of the infrared focal plane array and 29 an increase in operating temperature. Already established technologies in the mid infrared, such 30 as InSb or MCT or even promising detectors such as type II superlattices, achieve excellent 31 performances but fail to provide a low cost solution. In part, the high cost is due to the 32 requirement of epitaxial growth. $\mathrm{HgTe}$ CQD material can thus bring ease of processing and much 33 lower costs. ${ }^{15,16,17}$ Moreover the development of $\mathrm{HgTe}$ CQD benefits from the extensive 34 knowledge of $\mathrm{HgCdTe}$, making this material easily transferable to MCT foundry.

35 In this report we present an initial study of the temperature dependent conductivity and 36 photoconductivity characteristics of the HgTe CQD with mid-IR gaps. We report measurement of 37 the dark current, spectral response, responsivity and noise as a function of bias and temperature.

38 The efficiency of the CQD based detectors is evaluated and their optimal operating temperature identified. We identify possible pathways for optimizing the material as a photodetector. 


\section{EXPERIMENTAL}

\section{A. MATERIAL AND FILMS PREPARATION}

65 The synthesis of the QD particles uses the previously published method. ${ }^{1410}$ Briefly, $13 \mathrm{mg}$ of tellurium are dissolved in $2 \mathrm{~mL}$ of butanol and $0.1 \mathrm{~mL}$ of trioctylphosphine. The mixture is heated

67 to $90^{\circ} \mathrm{C}$ for 20 min under Ar to dissolve the tellurium. The temperature is then adjusted based on

68 the desired particle size, with higher temperatures leading to larger particles. The smallest

69 particles with band gaps reaching the visible region were obtained at temperatures as low as $0^{\circ} \mathrm{C}$

70 while the largest particles were prepared at $90^{\circ} \mathrm{C}$. Meanwhile mercury acetate is mixed with $2 \mathrm{~mL}$

71 of butanol and $2 \mathrm{~mL}$ of pyridine. This solution is then quickly injected into the flask, immediately

72 forming a dark solution. Finally the reaction is stopped after several minutes by extracting to a

73 room temperature solution of $10 \%$ dodecanethiol in tetracholoethylene (TCE).

74 The diameters of the dots in this study are in the 6 to $12 \mathrm{~nm}$ range. Particles with a $\sim 8 \mathrm{~nm}$ diameter

75 (respectively $10.5 \mathrm{~nm}$ ) present a band edge energy corresponding to $3 \mu \mathrm{m}(5 \mu \mathrm{m})$. Figure 1Figure

764 gives a transmission electron microscopy (TEM, Tecnai F30, 300kV) image showing

77 aggregated particles. Dynamic light scattering (DLS, Malvern, Zetasizer) measurements suggest

78 the nanocrystals are similarly aggregated in colloidal solution. The aggregate size distribution

79 follows approximately a log normal distribution typical, with a mean size which is typically 20

80 times larger than the single dot diameter. However the optical absorption shows confinement

81 which varies only with the dimensions of the individual particles in the aggregates.

82 Films of dots are obtained by drop-casting the TCE CQD solution on interdigitated Pt electrodes

83 on a glass substrate (ABTech IME 1050, 50 periods, 100nm thick, $10 \mu \mathrm{m}$ width and spacing). 
84 Films of HgTe CQD film studied here are a few hundred nanometers thick and we also 85 investigated thinner films made by using more dilute solutions. For all the following 86 measurements, the samples are mounted on the cold finger of a closed cycle He cryostat

87 (Coldedge SDRK 101D) operating between 3 and 300K. Three samples are under investigation in 88 this study with the following cut-off wavelength at room temperature $2.8 \mu \mathrm{m}$ (sample A), $3.4 \mu \mathrm{m}$

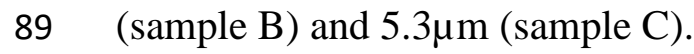

\section{B. EXPERIMENTAL SETUP}

$92 I(V)$ curves are measured using a Keithley 6487 pico-ammeter. The sample is illuminated by a 93 tungsten light-bulb. Its temperature, measured with a pyrometer, is adjusted in the 1400 to $942100^{\circ} \mathrm{C}$ range, giving an incident power of a few $\mathrm{mW}$ on the sample.

95 A home-made Michelson interferometer is used to measure spectral responses. A SiC lamp 96 (equivalent to a $1200^{\circ} \mathrm{C}$ blackbody) serves as broadband light source and is chopped at a

97 frequency in the $\mathrm{kHz}$ range. The detector signal is amplified using a Keithley 6487 and a PAR 98 model 124 lock-in.

99 The transient response of the detector is obtained by illuminating the sample with short pulses $100(10 \mathrm{ps}, 25 \mathrm{~Hz})$ of a $\mathrm{Nd}$ :YAG laser at $1.06 \mu \mathrm{m}$. The transient photocurrent is recorded using a 101 100MHz digital oscilloscope (Tektronix, TDS 1012B).

102 Noise measurements are performed with a spectrum analyzer (HP 3561a). The sample is biased 103 with a battery in order to limit the input noise. The mean signal is removed using a resistor bridge 104 and the noise current is then input to a low noise operational amplifier (LF356N, National 
120 Semiconductor). The current amplification is typically in the $10^{4}$ to $10^{7}$ range before input to the

121 spectrum analyzer. All the noise measurements were conducted in the cryostat with the optical 122 shutter closed.

\section{RESULTS AND DISCUSSIONS}

125

\section{A. AN INTRINSIC SEMICONDUCTOR}

127 Dark $I(V)$ curve for sample $\mathrm{C}$ are presented in Figure 2Figure 2. The dark $I(V)$ curves present

128 almost no hysteresis. They are generally linear at high temperature and become less linear as the

129 temperature decrease. The dark current as a function of temperature is also presented in Figure

130 2Figure 2. The transport decreases about two orders of magnitude from the room temperature

131 value, with an Arrhenius law $I(T) \propto \exp \left(-E_{a} / k_{b} T\right)$, with $E_{a}$ an activation energy, $k_{B}$ the

132 Boltzmann constant, and $T$ the temperature until settling to a lower slope. Activation energies are 133 reported in Table 1 Table 1 for sample A, B and C. 
136 Table 1: Activation energies for the dark current of the three samples. This value is compared to 137 the half value of the optical gap at room temperature. $N_{0}$ is the estimated random close packed 138 density of CQDs with the range based on a $20 \%$ standard deviation of the diameter. The carrier 139 density, $n$, is estimated based on a model for intrinsic thermal activation. .

\begin{tabular}{|c|c|c|c|c|c|c|c|c|c|}
\hline Sample & $\begin{array}{c}\lambda \text { cut-off } \\
(295 \\
\mathbf{K}) \\
(\mu \mathrm{m})\end{array}$ & $\begin{array}{c}\lambda_{\text {cut-off }} \\
(70 \mathrm{~K}) \\
(\mu \mathrm{m})\end{array}$ & $\begin{array}{c}E_{a} \\
(\mathrm{meV})\end{array}$ & $\begin{array}{c}\qquad \boldsymbol{E}_{G} / \mathbf{2} \\
(\mathbf{m e V}) \\
\text { From optical } \\
\text { measurement }\end{array}$ & $\begin{array}{c}N_{0} \\
\left(\times 10^{18}\right. \\
\left.\mathrm{cm}^{-3}\right)\end{array}$ & $\begin{array}{c}n \\
\left(\times 10^{16}\right. \\
\left.\mathrm{cm}^{-3}\right)\end{array}$ & $\begin{array}{c}\alpha \\
\left(10^{-4}\right. \\
\text { eV.K }\end{array}$ & $\begin{array}{c}\boldsymbol{\beta} \\
(\mathbf{K})\end{array}$ & $\begin{array}{c}\boldsymbol{E}_{\boldsymbol{G}}(\mathbf{0 K}) \\
(\mathbf{e V}) \\
\text { From } \\
\text { Varshni's } \\
\quad \text { fit } \\
\end{array}$ \\
\hline $\mathbf{A}$ & 2.8 & 3 & $\begin{array}{l}273 \\
\pm 10\end{array}$ & $\begin{array}{l}221 \\
\pm 44\end{array}$ & $2.6-7.6$ & $0.08-0.3$ & 1.5 & 500 & 0.43 \\
\hline B & 3.4 & 4.35 & $\begin{array}{r}201 \\
\pm 10 \\
\end{array}$ & $\begin{array}{r}182 \\
\pm 36\end{array}$ & $1.5-5$ & $0.2-0.8$ & 7 & 500 & 0.33 \\
\hline C & 5.3 & 6.6 & $\begin{array}{r}106 \\
\pm 10\end{array}$ & $\begin{array}{r}117 \\
\pm 20\end{array}$ & $0.6-2$ & $1-4$ & 7 & 500 & 0.23 \\
\hline
\end{tabular}

141 The activation energy is in each case close to half of the energy gap value (at room temperature),

142 the latter being determined as the edge of the photoresponse described below measured at room

143 temperature. Such a relation between the energy of thermal activation of carriers and the gap is

144 expected for an intrinsic semiconductor. At room temperature, we therefore conclude that the

145 carrier density is dominated by the intrinsic properties. There may of course be some carrier

146 doping in our samples, but given the thermal behavior of the dark current, it must be much less

147 that the density of intrinsic carriers at room temperature. In the intrinsic case, the carrier density

148 can be determined as $n=p=N_{0} \sqrt{N_{c} \cdot N_{v}} \exp \left(-E_{G} / 2 k_{B} T\right)$ where $N_{0}$ is the number of 149 nanocrystals per volume, and $N_{c}$ and $N_{v}$ are the numbers of electron and hole states respectively 150 in the nanocrystals that are thermally accessible. Given that much of the confinement energy is in 151 the electron states, we take $N_{c}=2$. For the more massive holes, we will also take $N_{v}=2$ even 
152 though it may be somewhat more. The estimated carrier densities at room temperature are then 153 shown in Table 1.

154 The measured resistivities of the samples allow an estimate of the mobility using $\rho^{-1}=n e \mu$, 155 which typically leads to value in the $0.5 \mathrm{~cm}^{2} \mathrm{~V}^{-1} \mathrm{~s}^{-1}$ range for sample $\mathrm{C}$. For the discussion later, 156 we also provide an estimate of the hopping time between nanocrystals. Using Einstein's relation,

157 the hopping time between nanocrystals and the mobilities are related by $\tau_{\text {hop }} \approx \frac{2 e R^{2}}{3 \mu k_{B} T} \approx 13 p s$ in 158 sample C. Hopping times faster than recombination times are helpful for efficient operation.

\section{B. EFFECT OF THE TEMPERATURE ON THE OPTICAL PROPERTIES}

161 Figure 3 shows the spectral response for the three samples. By changing the size and temperature 162 of the particles, the cut-off wavelength tunes from 2.8 to almost $7 \mu \mathrm{m}$. Shorter cut-off 163 wavelengths are accessible by growing smaller particles but are not in the scope of this work. In 164 samples B and C, some features in the spectral response are due to vibrational absorption of the $165 \mathrm{C}-\mathrm{H}$ bonds from the ligands $(3.5 \mu \mathrm{m}), \mathrm{H}_{2} \mathrm{O}(3 \mu \mathrm{m})$ and $\mathrm{CO}_{2}(4 \mu \mathrm{m})$ coming from water vapor and 166 carbon dioxide in the interferometer.

167 For all the samples, a decrease in temperature results in a red-shift of the spectral response, as 168 shown in Figure 3Figure 3and Table 1Table 1. This is consistent with bulk HgTe, where thermal 169 dilation of the HgTe lattice leads to a reduced gap with decreasing temperature. ${ }^{18,19}$ 
171 The thermal dependence of the energy gap is captured quite well with an empirical parabolic fit. 172 Alternatively, Varshni's ${ }^{20}$ expression, given by equation _(1)_ (1), is often used.

$173 E_{G}(T)=E_{G}(T=0 K)+\frac{\alpha T^{2}}{\beta+T}$

174 Here, the $\alpha$ parameter is linked to the thermal dilation coefficient. $\beta$ was originally associated 175 with the Debye temperature although it can deviate significantly ${ }^{2016}$. The parameter values for 176 bulk HgTe are poorly known and reported values cover a wide range, with $\alpha=2.7 \times 10^{-4} \mathrm{eV} \cdot \mathrm{K}^{-1}$ 177 to $8.5 \times 10^{-4} \mathrm{eV} \cdot \mathrm{K}^{-1} . \underline{1814,1915}$ Our experimental points are compared with a Varshni's law fit for 178 the thermal dependence. As shown in Figure 2Figure 2, the fit is imperfect and is in fact not very 179 sensitive to the choice of $\beta$, set at $500 \mathrm{~K}$, but the coefficient $\alpha$ is reasonable. Table 1 lists the 180 parameters. With more monodispersed samples it will be important to determine these parameters 181 with more accuracy than in the bulk and to extract their size dependence ${ }^{21}$ since the red shift with 182 temperature is relevant to the mid-IR detection.

183 Increasing the bias increases the magnitude of the response but it does not change its spectral 184 shape. The relative responsivity as a function of temperature is shown in Figure $4 \mathrm{~b}$ and is 185 obtained by the integral of the photocurrent spectrum. At sufficiently high temperature, the 186 responsivity reaches a regime where it is independent of the detector temperature. However, at a 187 low enough temperature, the responsivity decreases exponentially.

188 The responsivity is proportional to the exciton ionization efficiency of a single dot multiplied by 189 the gain over the structure which is the ratio of the carrier lifetime over the carrier transit time, ${ }^{22}$ 190 as given by equation $\underline{(2(2))}$. 
$191 \quad R \propto \eta \frac{\tau_{L T}}{\tau_{\text {transit }}} \quad(2)$

192

Future measurements of each of these parameters will be needed. At present we speculate that the 193 ratio of carrier lifetime and transit times is a constant independent of temperature and bias. This 194 195 196 197 will be the case if carrier trapping plays a significant role. A possible example of traps could be very large dots with therefore vanishing gaps. In this case, the major contributor to the temperature variation of $R$ is the ionization efficiency $\eta$. We then consider that the ionization rate is thermally activated $k_{I}=k_{I 0} \exp \left(-E / k_{b} T\right)$ and competes with a fixed recombination rate $k_{r}$ 198

due to radiative and nonradiative processes within one dot. The ionization efficiency is then $\eta_{\text {single QD }}=\frac{e^{-E_{I} / k T}}{k_{r} / k_{I o}+e^{-E_{I} / k T}}(3)$

200 Fitting the experimental data with such an expression leads to a strong discrepancy at the lowest temperature since it predicts $\eta=0$ at $0 \mathrm{~K}$. Such a deviation may result from the distribution of activation energies. For a collection of dots, we choose a Gaussian energy distribution such that

$$
\eta_{\text {ensemble }}\left(T, E_{i}, \sigma\right)=\int_{-\infty}^{\infty} \eta_{\text {single QD }}(T, E) \frac{1}{\sigma \sqrt{2 \pi}} \exp \left(-\frac{\left(E-E_{i}\right)^{2}}{2 \sigma^{2}}\right) d E
$$

204 This provides a better fit with values of the mean activation energies of $160 \mathrm{meV}$ for sample A, $205100 \mathrm{meV}$ for sample B and $34 \mathrm{meV}$ for sample $\mathrm{C}$. The broadening of the ionization energies are 206 within $20-50 \%$. In this model, the saturation of the ionization efficiency at moderate temperature 207 implies that the time required for charge separation becomes smaller than the recombination time, 208 radiative and non-radiative, and therefore that the quantum yield of charge generation is already 
209 close to unity. This is compatible with the fast hopping time mentioned earlier at room 210 temperature.

211 The origin of these activation energies for charge separation may lie in the disorder associated 212 with the size inhomogeneity and in an energy barrier associated with charge transfer, even in the 213 absence of disorder. To estimate the latter, we use Marcus theory ${ }^{23}$ considering that the electron 214 (or hole) transfers from an exciton in a sphere with binding energy $E_{e x}$ to a neighboring sphere.

215 The Exciton binding energy is given by ${ }^{24} E_{e x}=1.8 \frac{e^{2}}{\varepsilon R}$, with $R$ the radius of the dot and $\varepsilon$ their 216 dielectric constant. Assuming a value of $\varepsilon=20$ in $\mathrm{HgTe}^{25,26}$, this is estimated as $32 \mathrm{meV}$ and 24 $217 \mathrm{meV}$ for samples $\mathrm{B}(R=4 \mathrm{~nm})$ and $\mathrm{C}(R=5.3 \mathrm{~nm})$. The Marcus expression for the energy barrier is $218 \Delta G_{0}=\frac{\lambda_{0}}{4}\left(1+\frac{E_{e x}}{\lambda_{0}}\right)^{2}$ where $\lambda_{0}$ is the reorganization energy. For electron transfer in a two-sphere 219 model $\lambda_{0}=e^{2}\left(\frac{1}{R}-\frac{1}{R+\delta}\right)\left(\frac{1}{\varepsilon_{\infty}}-\frac{1}{\varepsilon_{0}}\right)$,where $R$ is the dot radius, and $\delta$ is the matrix thickness 220 between the dot surfaces estimated as $\sim 1 \mathrm{~nm}$ for the dodecanethiol. The dynamic and static 221 dielectric constants, $\varepsilon_{\infty}$ and $\varepsilon_{0}$, refer to the matrix. This is unknown for the matrix material, 222 mostly bound alkane thiol, but we take $\varepsilon_{\infty}=2.1<<\varepsilon_{0}$. This gives energy barriers of $32 \mathrm{meV}$ and $22325 \mathrm{meV}$ for samples B and C respectively. Energy disorder due to size inhomogeneity could 224 easily be a larger effect, and may explain the discrepancy with measured values above.

\section{RESPONSIVITY}

227 Using the $I(V)$ curves under illumination, as described in reference 1410 , we can extract the absolute responsivity of our detectors. To proceed, we first identify a temperature at which the 
246 dark current is far below the photocurrent in our range of flux (room temperature for the A 247 sample, $190 \mathrm{~K}$ for the $\mathrm{C}$ sample). We then measure two $I(V)$ curves under illumination at this 248 temperature, for two different amounts of incident flux. This latter is evaluated as in equation

$250 \phi\left(T_{B B}, \alpha, \beta\right)=A_{d} \pi \cdot \cos \beta \cdot \sin ^{2} \alpha \int_{\lambda \min }^{\lambda_{\text {cutoff }}} \frac{2 h c^{2}}{\lambda^{5}} \frac{1}{e^{\frac{h c}{\lambda k T_{B B}}}-1} d \lambda$,

251 Where $A_{d}$ is the active area of the sample $\left(0.1 \mathrm{~cm}^{2}\right), \alpha$ is the half angle of view of the detector and $252 \beta$ is the angle between the normal of the detector and the incident light, $h$ is the Planck constant, $c$ 253 the speed of the light, and $T_{B B}$ the equivalent black-body temperature of the light source.

254 Finally the responsivity is given by equation $(4)-(4)$

$255 R=\frac{I_{\text {total }}\left(\phi_{1}\right)-I_{\text {total }}\left(\phi_{2}\right)}{\phi_{1}-\phi_{2}}$

256 We thus obtained the absolute responsivity at one temperature and the relative responsivity in

257 Figure 4Figure 4 (b), for the other temperatures. Finally the responsivity as a function of the 258 applied bias is plotted in Figure 5Figure 5. The responsivity appears linear with bias.

\section{NOISE AND DETECTIVITY}

260 The spectrum analyzer provides the voltage noise density $\left(v_{n}\right)$. The current noise density $\left(i_{n}\right)$

261 which is the useful quantity to evaluate the detectivity, is then obtained by $i_{n}=\frac{v_{n}}{G \cdot B W}$, where $G$ 262 is the current amplification and $B W$ is the bandwidth of the spectral acquisition. 
283 The noise for sample $\mathrm{C}$ is plotted in Figure 6Figure 6 (a) and (b). At all biases and temperatures,

284 a non-white noise prevails at low frequency, and follows a power law with an exponent in the -

2850.4 to -0.7 range consistent with $1 / f$ noise. As temperature and bias increase, the dark current and

286 the noise both increase.

$2881 / f$ noise can be modeled using the Hooge's law ${ }^{27}$,

$289 \quad i_{1 / f}^{2}=\frac{\alpha_{H} \cdot I^{2}}{N \cdot f}$

290 where $N$ is the number of carriers, $I$ the average current through the sample, $f$ the frequency and $291 \alpha_{H}$ is a parameter which describes the magnitude of the $1 / f$ noise. Hooge reported that in most 292 metallic materials, this parameter is rather close to $2 \times 10^{-3}$. While we do not know of reported 293 values of $\alpha_{H}$ for bulk $\mathrm{HgTe}, \alpha_{H}$ has a value in the $10^{-3}$ to $10^{-5}$ range for $\mathrm{HgCdTe}$ detectors ${ }^{28,29}$ 294 (with a cadmium content in in the 0.17 to 0.3 range), also much smaller than observed here. We 295 extract $\frac{\alpha_{H}}{N}=f \frac{i_{1 / f}^{2}}{I^{2}}$, shown in Figure 6Figure 6 (c) for $\mathrm{f}=10 \mathrm{~Hz}$. The number of carriers can be 296 evaluated as the product of the carrier density, which was estimated in table 1, and the volume of 297 the sample. This gives $4 \times 10^{10}$ carriers for sample C. Since the value of $\frac{\alpha_{H}}{N}$ is typically $10^{-11}$ for 298 sample $\mathrm{C}, \alpha_{H}$ is therefore around 0.4, two decades above the Hooge value. The larger value of 299 the Hooge constant therefore suggests that our CQD samples are possibly about 10 fold noisier 300 than they need to be. One possibility is that the sample inhomogeneity induces current 301 crowding ${ }^{30,31}$ such that the actual number of carriers should not be related to the total volume of 302 the sample. Whether the noise level is intrinsically related to the nanoparticle assembly or more 
323 specific of the particular samples that we have studied, and in particular the aggregation

324 discussed in section I, Figure 1, remains unknown and is a target of further study.

325 Finally, by combining the responsivity and noise measurements we evaluate the specific

326 detectivity of the samples, using equation $\underline{(6)(6)}$.

327

$D^{*}=\frac{R \sqrt{A_{d}}}{i_{n}}$

Maps of the detectivity as a function of the operating temperature and applied bias are presented

329

in Figure 7Figure 7.

Mis en fo

330 These maps show that the HgTe CQD material is able to reach detectivity above $10^{9}$ Jones. The

331 optimal operating temperature is just below room temperature $(270 \mathrm{~K})$ for sample A $\left(\lambda_{\text {cut-off }}=\right.$ $3322.8 \mu \mathrm{m}$ ) and is around $130 \mathrm{~K}$ for sample $\mathrm{C}$ (with a $\lambda_{\text {cut-off }} \approx 6 \mu \mathrm{m}$ at this temperature). The applied 333 optimal biases are respectively for sample A and C $19 \mathrm{~V}$ (corresponding to an electric field of $\left.3348 \mathrm{kV} \mathrm{cm}^{-1}\right)$ and $8 \mathrm{~V}\left(19 \mathrm{kV} \mathrm{cm}^{-1}\right)$, which is above the applied bias of the regular read-out circuit 335 (generally a few volts). Nevertheless this optimal bias can easily be reduced by changing the 336 spacing of the electrodes.

\section{E. INFLUENCE OF THE THICKNESS}

The thickness of the CQD film is an important parameter determining some of the detector properties. In particular, based on bulk optical properties of $\mathrm{HgTe}$, the absorption depth of films of CQDs is expected to be around 5 microns, which is an order of magnitude larger than the thickest samples studied. Correspondingly, the optical densities of the samples were rather small, 
of the order of 0.1 . Thicker films will directly lead to improved responsivities. While making

358 uniform thick films will require a dedicated effort, it is interesting to explore how the response of

359 thinner films varies with thickness. Therefore, three samples based on sample $\mathrm{C}$ of respective 360 thickness 15, 35 and 450nm have been studied. Their properties are summarized in Table 2Table

$361 Z$. The thickness is measured using an Atomic Force Microscope, scanning a groove made with a 362 sharp razor blade.

363 Table 2: Properties of the three films based on sample $C\left(\lambda_{\text {cut-off }} \approx 5 \mu \mathrm{m}\right)$ and of different 364 thicknesses. The time response is the 1/e decay time.

\begin{tabular}{cccc}
\hline \hline & $\begin{array}{c}\text { Thick } \\
\text { film }\end{array}$ & Medium film & Thin film \\
\hline Appearance & Brown & $\begin{array}{c}\text { Barely } \\
\text { colored }\end{array}$ & Colorless \\
\hline $\begin{array}{c}\text { Thickness } \\
\text { (nm) }\end{array}$ & 450 & 35 & 15 \\
\hline $\begin{array}{c}\text { Dark Current, } \\
\text { at 10V (mA) }\end{array}$ & 22 & 0.56 & 0.031 \\
\hline $\begin{array}{c}\text { Resistance } \\
\text { (room T) } \\
\text { at 10V (k } \Omega)\end{array}$ & 0.5 & 17 & 320 \\
\hline $\begin{array}{c}\text { Responsivity } \\
\text { (normalized } \\
\text { by thick film } \\
\text { value) }\end{array}$ & 1 & $7 \%$ & $0.2 \%$ \\
\hline $\begin{array}{c}\text { Decay time } \\
\text { (ns) }\end{array}$ & 63 & $2 \times 10^{3}$ & $5 \times 10^{5}$ \\
\hline \hline
\end{tabular}

366 Even the thinnest film provides a measurable mid-IR photoresponse, while being very transparent 367 and only a couple layers of dots. Generally the thicker the film the smaller the resistance, see 368 Figure 8Figure 8 (a). An increase of the thickness also results in greater absorption which leads to 369 a higher responsivity (Figure 8Figure 8(c)). In parallel, the noise also increases with sample 370 thickness. According to Hooge's expression, we expect the current $1 / f$ noise to increase as the 
square root of thickness while the responsivity increases linearly with the thickness as long as it

394 remains much smaller than the absorption length. Therefore, there should be a net increase of the

395 detectivity with square root of the thickness. A square root fit is then used as a guide to the eye in $396 \quad$ Figure 8Figure 8 (e).

397 The measurement of the transient photocurrent has been used to probe the density of states inside 398 the gap of several materials. ${ }^{32,33}$ Here, we focus on the transient photocurrent decay, which is 399 related to the cut-off frequency of the detector. Surprisingly, an increase in film thickness gives a 400 faster response of the detector, see Figure 8Figure 8 (b). It is also observed that an increase of the

401 bias leads to a faster response, both for the rise and decay time while the incident beam energy 402 only slightly affects the transient shape. Increasing the operating temperature of the detector, 403 from $250 \mathrm{~K}$ to $295 \mathrm{~K}$, also leads to a faster response. While these effects are not completely 404 understood, they likely arise from capacitance associated with space charge at the contacts ${ }^{34}$. 405 While this capacitance is constant for a given electrode geometry, the resistance of the devices 406 drops with increasing film thickness, bias and temperature, leading to a decreased RC time 407 constant. Compared to $\mathrm{PbS}$ CQD detector ${ }^{9}$ operating in the near-IR, the HgTe times response are 408 much faster but the responsivities are also lower, in accordance with equation $\underline{(2(z)) .}$

410 Since the optimal detectors need to have thicknesses larger than the thickest one studied here in 411 order to saturate the absorption, we expect that the speed of the detector will be much faster than 412 required for usual imagery application for which the typical integration time is in the $1 \mathrm{~ms}$ range. 
415 The conductivity and mid-infrared response photoconductivity of HgTe CQD solid films are 416 investigated as a function of temperature. By changing the size of the particle and the 417 temperature, the cut-off wavelength has been tuned from the near infrared up to $7 \mu \mathrm{m}$. The 418 measured responsivity already competes with existing devices such as QWIP ${ }^{35}$ or type II 419 superlattices ${ }^{36,37}$ and further improvements are expected with optimized thicker films.

420 The dark current is thermally activated with an activation energy close to half of the energy gap.

421 This suggests that, at room temperature, most carriers are thermally generated. From the 422 estimated carrier density, the mobility is extracted and is relatively large at room temperature, of 423 the order of $0.1-1 \mathrm{~cm}^{2} \mathrm{~V}^{-1} \mathrm{~s}^{-1}$, especially given that the material is a simple drop-cast film of CQDs 424 without any attempt to eliminate or replace the ligands. These mobility values will need to be 425 confirmed by direct measurements. We observe that the temperature dependence of the 426 responsivity is much weaker than that of the dark current, being essentially constant around room 427 temperature and dropping only at lower temperatures. We interpret this behavior as arising from 428 already efficient charge separation at room temperature. The films show $1 / \mathrm{f}$ noise at low 429 frequency with values of the Hooge's constant that are two orders of magnitude larger than for 430 clean homogeneous metals, although the noise decreases with temperature leading to an optimum 431 operating temperature. The best detectivity values for a $450 \mathrm{~nm}$ thick film are $\sim 2 \times 10^{9}$ jones at $432130 \mathrm{~K}$ and the fastest response times are below $100 \mathrm{~ns}$ at room temperature. We stress again that 433 these results have been obtained with no processing of the films other than simply drop-cast from 434 solutions.

435 Since the mobility and carrier generation efficiency are already high it seems that further 436 improvement will rather come from the improved optical absorption edge, longer carrier lifetime, 437 and reduced noise. Improved optical properties come from thicker films, and materials with 
438 improved monodispersivity. Higher monodispersivity will also reduce the concentration of large 439 dots which may act as recombination centers. Reducing the noise will involve investigating the 440 effect of film processing to possibly reduce the crowding effect which maybe the source of 441 excess $1 / f$ noise. It is expected that detectivities above $10^{10}$ Jones in the mid-IR will be achievable 442 while maintaining a fast response time and moderate cooling temperatures consistent with 443 imaging operation.

$445 \quad$ V. ACKNOWLEDGEMENTS

446 The research was supported by the US National Science Foundation NSF under Grant No 447 DMR-070626 and by DOE under Grant No. DE-FG02- 06ER46326. The authors made use of 448 shared facilities supported by the NSF MRSEC Program under DMR-0820054. EL thanks Ecole 449 Polytechnique, Palaiseau, France, for a postdoctoral fellowship. 
452 Figure 1: (a) TEM image of the HgTe material. (sample C) Scale bar is $10 \mathrm{~nm}$. The inset shows a 453 high resolution image of a single dot. (b) Diameter distribution of single dots for sample C 454 measured from TEM imaging (c) Hydrodynamic radii distribution in solution (sample C) 455 obtained by dynamic light scattering (DLS), and a log normal fit of the distribution with a 220 456 nm mean value.

457

458 Figure 2: Dark current for sample C, as a function of the inverse of the temperature, for three 459 different biases. The inset shows the $I(V)$ curve for the sample $\mathrm{C}$ for two different temperatures.

461 Figure 3: Normalized spectral response for the sample A (top), B (middle) and C (bottom) for 462 different temperatures. These spectra have been acquired while the samples were respectively 463 biased by $2 \mathrm{~V}, 1 \mathrm{~V}$ and $0.3 \mathrm{~V}$.

465 Figure 4 (a) Position of the long-wavelength half maximum of the spectral response as a function 466 of the temperature for the sample C. (b) Relative responsivity (integral of the photocurrent 467 spectrum), normalized to the room temperature value as a function of temperature for samples A, 468 B and C. The solid lines are associated fit as discussed in the text. 
470 Figure 5: Responsivity for samples A, B and C as a function of the applied bias for different 471 temperatures.

473 Figure 6: (a) Current noise spectral density for a 2V bias at temperatures: 70, 110, 150, 190, 230, 474250 and $295 \mathrm{~K}$ for sample C. (b) Current noise spectral density at 250K at applied bias: 0.5, 2 and $4755 \mathrm{~V}$ for sample C. (c) Ratio of the $\alpha_{\mathrm{H}}$ Hooge's parameter over the number of carriers as a function 476 of the detector temperature at $10 \mathrm{~V}$ for sample $\mathrm{C}$ at $10 \mathrm{~Hz}$.

477

478 Figure 7: Map of the specific detectivity as a function of temperature and applied bias for sample 479 A (top) and C (bottom).

481 Figure 8: (a) Dark current as a function of the applied voltage for three films. (b) Transient 482 photocurrent in response to a short (10ps) laser pulse of $1.06 \mu \mathrm{m}$ wavelength, with $7 \mathrm{~V}$ bias (c) 483 Relative responsivity as a function of the applied bias, (d) noise current spectral density, at a 7V 484 bias and (e) Detectivity (at 7V) as a function of film thickness for three films of different 485 thicknesses based on the material of sample C. All measurements were conducted at room 486 temperature. In the graphs a, b, c, d, the solid line is for the $15 \mathrm{~nm}$ film, the dashed line for the $48735 \mathrm{~nm}$ film and the dotted line for the 450nm film. 
${ }^{1}$ For a recent review see D. Talapin, J.S. Lee, M. Kovalenko and E. Shevchenko, Chem. Rev. 110, 389 (2010).

${ }^{2}$ G.I. Koleilat, L. Levina, H. Shukla, S.H. Myrskog, S. Hinds, A.G. Pattantyus-Abraham, E.H. Sargent, ACS Nano. 2, 833 (2008).

${ }^{3}$ J. Tang, E. H. Sargent, Advanced Materials 23, 12 (2011).

${ }^{4}$ I. M Tsidilkovski, Electron spectrum of gapless semiconductors, Spinger series on solid state sciences 116, Springer, Heidelberg, 1997.

${ }^{5}$ G. Nimtz and B. Schlicht, Narrow-gap semiconductors, Springer tracts in modern physics 98 , Spinger, Heidelberg, 1983.

6 T. Rauch, M. Böberl, S. F. Tedde, J. Fürst, M. V. Kovalenko, G. Hesser, U. Lemmer, W. Heiss, O. Hayden, Nature Photonics 3, 332 (2009)

${ }^{7}$ E. H. Sargent, Adv. Mater. 17, 515 (2005).

${ }^{8}$ G. Konstantatos, C. Huang, L. Levina, Z. Lu and E. H. Sargent, Adv. Func. Mater. 15, 1865 (2005).

${ }^{9}$ G. Konstantatos, I. Howard, A. Fischer, S. Hoogland, J. Clifford, E. Klem, L. Levina and E. H. Sargent, Nature 442, 180 (2006).

10 J. M. Pietryga, R. D. Schaller, D. Werder, M. H. Stewart, V. I. Klimov, and J. A. Hollingsworth, J. Am. Chem. Soc. 126, 11752 (2004).

${ }^{11}$ I. M Tsidilkovski, Electron spectrum of gapless semiconductors, Spinger series on solid state sciences 116, Springer, Heidelberg, 1997.

12 M. Kovalenko, E. Kaufmann, D. Pachinger, J. Roither, M. Huber, J. Stangl, G. Hesser, F. Schaffler, and W. Heiss, J. Am. Chem. Soc. 128, 3516 (2006).

${ }^{13}$ M. Böberl, M. V. Kovalenko, S. Gamerith, E. J. W. List, and W. Heiss, Advanced Materials 19, 3574 (2007).

${ }^{14}$ S. Keuleyan, E. Lhuillier, V. Brajuskovic and P. Guyot-Sionnest, Mid-infrared HgTe colloidal quantum dot photodetectors, submitted to Nat. Photonics (2011).

${ }^{15}$ L. Kim, P. O. Anikeeva, S. A. Coe-Sullivan, J. S. Steckel, M. G. Bawendi and V. Bulovic, Nano Lett 8, 4513 (2008).

16 V. Wood, M. J. Panzer, J. Chen, M. S. Bradley, Jonathan E. Halpert, M. G. Bawendi, V. Bulović, Advanced Materials 21, 2151 (2009).

${ }^{17}$ T. Kim, K.Cho, E. K. Lee, S. J. Lee, J. Chae, D. H. Kim, J. Kwon, G. Amaratunga, S. Y. Lee, J. Kim, B. L. Choi, Y. Kuk, J. M. Kim and K. Kim, Nat. Photonic 5. 176 (2011).

${ }^{18}$ C. S. Guenzer and A. Bienenstock, Phys. Rev. B 8, 4655 (1973).

${ }^{19}$ M. Dobrowolska, A. Mycielski and W. Dobrowolski, Solid State Comm. 27, 1233 (1978).

${ }^{20}$ Y. P. Varshni, Physica 34, 149 (1967).

${ }^{21}$ A. Olkhovets, R.-C. Hsu, A. Lipovskii, and F. W. Wise, Phys. Rev. Lett. 81, 3539 (1998).

22 E. Rosencher and B. Vinter, in Optoelectronics (Cambridge University Press, Cambridge, 2002).

${ }^{23}$ R.A. Marcus, Pure and Appl. Chem. 69, 13 (1997)

${ }^{24}$ L. E. Brus, J. Chem. Phys. 79, 5566, (1983). 
25 K. Ortner, X. C. Zhang, A. Pfeuffer-Jeschke, C. R. Becker, G. Landwehr, and L. W. Molenkamp, Phys. Rev. B 66, 075322 (2002).

${ }^{26}$ V. Radhakrishnan and P. C. Sharma, Phys. Rev. B 23, 3004 (1981).

${ }^{27}$ F. N. Hooge, IEEE Trans. Elec. Devices 41, 1926 (1994).

28 R.J. Westerhout, C.A. Musca, J. Antoszewski, J.M. Dell and L. Faraonen, J. Electronic Materials 36, 884 (2007).

${ }^{29}$ C.T. Elliot, N.T. Gordon, R.S. Hall, and T.J. Phillips, C.L. Jones and A. Best, J. Electronic Materials 26, 643 (1997).

${ }^{30}$ E.P. Vandamme and L.K.J. Vandamme, Microelectronics reliability 40, 1847 (2000).

31 A. Mercha, L.K.J. Vandamme, L Pichon, R carin and O. Bonnaud, J. Appl. Phys. 90, 4019 (2001).

33

A. Rose, Phys. Rev. 97, 1538 (1954).

${ }^{35}$ V. Guériaux, A. Nedelcu and P. Bois, J. Appl. Phys. 105, 114515 (2009).

${ }^{36}$ E. Plis, J.B. Rodriguez, G. Balakrishnan, Y.D. Sharma, H.S. Kim, T. Rotter and S. Krishna, Semicond. Sci. Technol. 25, 085010 (2010).

${ }^{37}$ M. Walther, R. Rehm, F. Fuchs, J. Schmitz, J. Fleissner, W. Cabanski, D. Eich, M. Finck, W. Rode, J. Wendler,R. Wollrab and J. Ziegler, J. Elec. Mat. 34, 722 (2005). 